\title{
Buchbesprechung
}

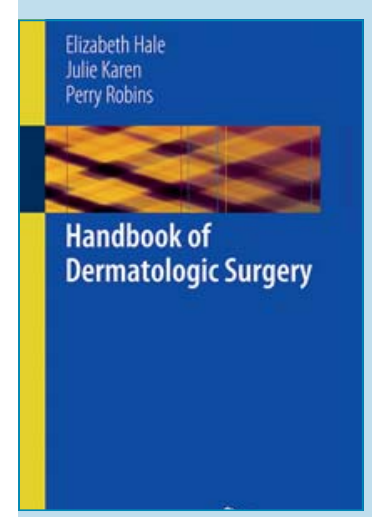

Es beginnt mit einem Kapitel über anatomische Gegebenheiten, vor allem im Gesicht, darauf folgt ein langes Kapitel mit der bildlichen und schriftlichen Darstellung häufiger Lappenplastiken und Nahttechniken sowie der tabellarischen Aufzählung der verschiedenen Lokalanästhetika, Desinfektionsmittel und Fadenmaterialen mit ihren jeweiligen Einsatzgebieten und Vor- und Nachteilen. Auch auf OP-Komplikationen wird eingegangen. Außerdem werden die verschiedenen OP-Instrumente grafisch dargestellt und benannt.

\section{Handbook of Dermatologic Surgery}

Hale E, Karen J, Robins P (Hrsg)

ISBN 978-1-4614-8334-2
Dieses kleine Taschenbuch aus dem Springer Verlag gibt einen raschen Überblick über gängige dermatologische OP-Techniken sowie die Lasertherapie und ästhetisch-kosmetische Verfahren.

Berlin/Heidelberg: Springer, 2014, 126 S., 38 Abb., 74,89€

Im nächsten Kapitel werden schwierigere und seltenere Lappenplastiken sowie Transplantate dargestellt und mit ihren Indikationen sowie den Vor- und Nachteilen beschrieben. Im 4. Kapitel werden wiederum in tabellarischer Form die verschiedenen Filler, Neurotoxine und chemischen Peelings mit den entsprechenden Einsatzgebieten aufgelistet. In den letzten 2 Kapiteln werden die verschiedenen Lasersysteme mit Indikationen genannt sowie die Möglichkeiten der Venenbehandlung mittels Laser- und Sklerotherapie.

Das Pocket-Buch richtet sich an Studenten, Assistenzärzte und Fachärzte im Bereich der operativen Dermatologie und dient als schnelles Nachschlagewerk z.B. im OP. Wen es nicht stört, dass es bisher nur in englischer Sprache vorliegt, sei dieses Buch empfohlen, auch wenn die Anschaffungskosten recht hoch sind.

Dr. med. Ines Hüsemann, Wiesbaden 